\title{
Birefringence as a Mechanism for the Broadening and Depolarization of Pulsar Average Profiles
}

\author{
M. M. McKinnon \\ NRAO, Green Bank, WV, USA
}

\section{Introduction}

Shortly after orthogonal modes of polarization were observed in pulsar radio emission (Manchester et al. 1975), a series of theoretical papers (Cocke \& $\mathrm{Pa}$ cholczyk 1976, Melrose \& Stoneham 1977, Melrose 1979) were quick to point out that these abrupt, 90 degree transitions in polarization position angle could be explained by a propagation effect. More specifically, the plasma above pulsar polar caps may be birefringent. The orthogonal modes separate because they have different indices of refraction. So, as Allen \& Melrose (1982) point out, the plasma is somewhat analogous to a terrestrial birefringent crystal, at least in the way it affects the ray paths of the modes. With very sensitive observations of individual pulse polarization, Stinebring et al. (1984) concluded that orthogonal modes are superposed, meaning they occur simultaneously. This observation is consistent with the birefringence hypothesis. Stinebring et al. also suggested that pulsars depolarize at high radio frequency via the superposed modes. Barnard \& Arons (1986) expanded on the earlier theoretical work, and proposed that, in addition to orthogonal modes, birefringence could account for the broadening of pulsar average profiles at low radio frequency and their depolarization at high frequency. The purpose of this paper is to further investigate the suggestions of Stinebring et al. and Barnard \& Arons.

\section{Broadening and Depolarization Due to Birefringence}

First of all, how does birefringence broaden and depolarize a pulse? Using the analogy of Allen \& Melrose, consider an unpolarized light beam which is shining on a calcite crystal. Calcite is birefringent because the ordinary $(O)$ and extraordinary $(\mathrm{X})$ wave propagation modes have different indices of refraction in the crystal. The index of refraction of the $\mathrm{O}$-mode is independent of the orientation of the beam with respect to the optic axis of the crystal. However, the index of refraction of the $\mathrm{X}$-mode is orientation-dependent. When the incident beam is parallel to the crystal optic axis, the indices of refraction of the $\mathrm{X}$ and O-modes are the same $(n \simeq 1.5)$. But when the incident beam is perpendicular to the optic axis, the $\mathrm{X}$-mode has an index refraction of $n_{x} \simeq 1$. The difference in the indices of refraction causes the $\mathrm{X}$ and $\mathrm{O}$-mode beams to separate as they exit the crystal. Since the modes are completely and orthogonally-polarized, the crystal performs the function of a polarizing beam splitter.

Next, how do the overall size of the beam that exits the crystal and its polarization vary as the orientation of the incident beam is changed with respect to 
the crystal optic axis? The $\mathrm{X}$ and $\mathrm{O}$-mode beams attain their largest separation when the incident beam is perpendicular to the crystal optic axis. The overall size of the exit beam is large and its polarization is complete. If the angle between the incident beam and the optic axis is decreased slightly, the separation between the individual modes decreases, and, consequently, the overall size of the exit beam decreases. Since the $\mathrm{X}$ and $\mathrm{O}$-mode beams do not overlap, the polarization of the exit beam is still complete. However, at some critical angle where the incident beam is nearly parallel to the optic axis, the $\mathrm{X}$ and $\mathrm{O}$-mode beams will begin to overlap, and the exit beam will begin to depolarize. The depolarization occurs while the overall size of the exit beam is relatively constant. Recall that above the critical angle, it is the polarization that remains constant while the overall size decreases. Also bear in mind that the broadening and the depolarization of the exit beam are the result of a propagation effect. They are independent of the emission mechanism (the intensity of the incident light beam).

\section{Spectra of Linear Polarization and Pulse Width}

For wave propagation in the flux tube plasma above a pulsar's polar cap, it is the frequency dependence of the indices of refraction that causes the modes to separate, not the orientation of the plasma "optic axis" with respect to the emission region. Additionally, the details of the mode separation may be slightly different. In the model of Barnard \& Arons, the plasma is transparent to the $\mathrm{X}$-mode, and the ray paths of the $\mathrm{X}$-mode are parallel to the magnetic axis of the pulsar. The O-mode rays, however, are bent or "ducted" along the magnetic field lines. Although the details of the mode separation are different, the broadening and depolarization of the pulse should be generally similar to that discussed above.

The spectra of flux density, fractional linear polarization, and pulse width for PSR B1133+16 are shown in Figure 1. References for the flux density, polarization, and pulse width data are given in Malofeev \& Malov (1980), Morris et al. (1981), and Thorsett (1991), respectively. The vertical dashed line in each spectrum indicates a critical or break frequency as determined from independent study (Malofeev \& Malov 1980, Morris et al. 1981, and Backer 1976). Similar to the broadening and depolarization by a birefringent crystal as demonstrated in the preceding section, the pulse depolarizes while the width remains constant above the critical frequency. Below the critical frequency, the width varies and the polarization remains constant. The spectra of PSRs B0823+26 and B0950+08 behave in a similar way (McKinnon 1996). In the case of PSR $\mathrm{B} 0950+08$, the pulsar depolarizes rapidly above its critical frequency of $200 \mathrm{MHz}$ (Morris et al. 1981). The fact that its pulse profile actually bifurcates near 200 $\mathrm{MHz}$ (Phillips \& Wolszczan 1992) makes the birefringence scenario even more tantalizing (McKinnon 1996).

\section{Rates of Depolarization}

If pulsars depolarize via superposed orthogonal modes, then why do pulsars have different rates of depolarization? When the orthogonal modes are superposed, 

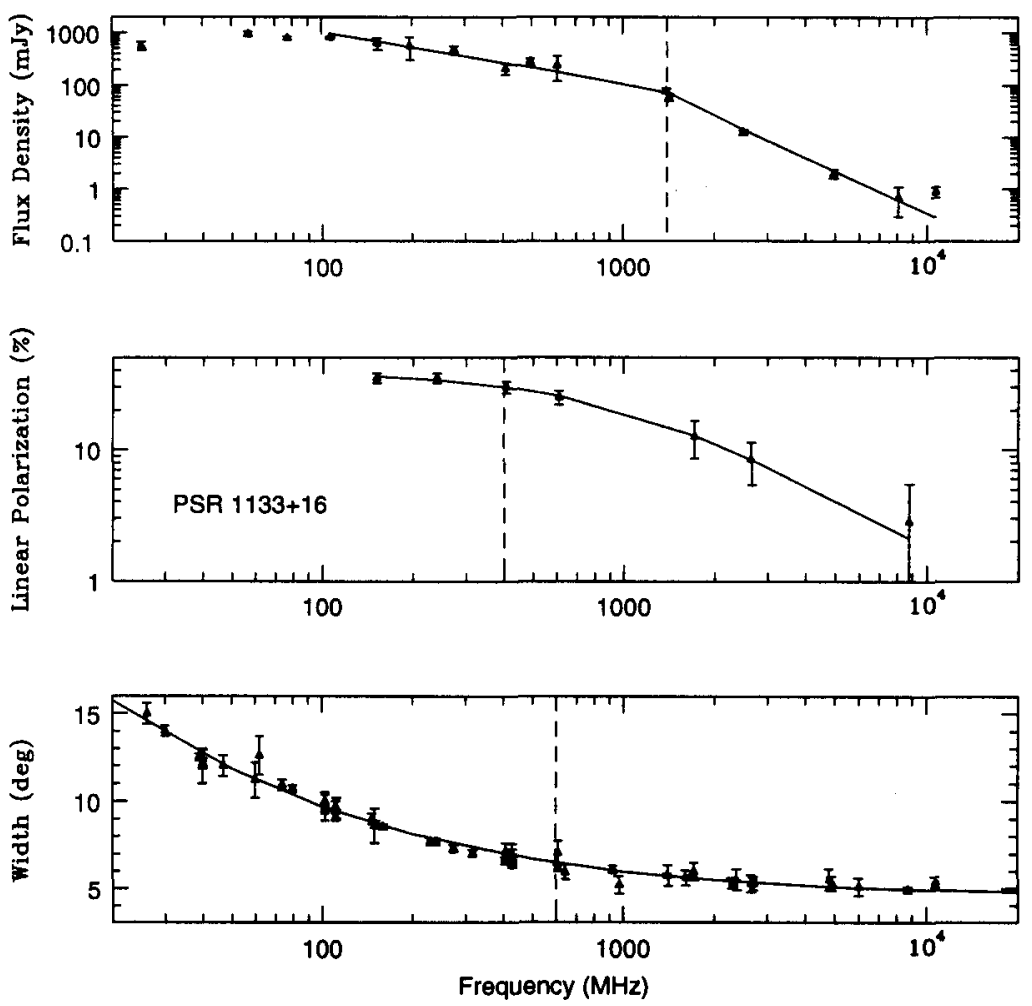

Figure 1. Spectra of total intensity, fractional linear polarization, and pulse width for PSR B1133+16. The critical frequency in each spectrum is identified by a dashed line.

the instantaneous polarization is related to the difference between the polarizations of the two modes, $L=\left|L_{1}-L_{2}\right|$. The instantaneous position angle is that of the stronger mode. The switching between orthogonal modes appears to be related to some type of stochastic process, so let the polarization of the modes be distributed according to the functions $f_{1}\left(\mu_{1}, \sigma_{1}\right)$ and $f_{2}\left(\mu_{2}, \sigma_{2}\right)$. The distributions have means, $\mu$, and standard deviations, $\sigma$. The means are generally not equal because the frequency of occurrence of the modes is not the same in the observed distributions of polarization position angle. When the means are nearly equal, the instantaneous polarization will generally be small, suggesting that the pulsar will depolarize rapidly. But if the means are quite different, the instantaneous polarization can be large, and the rate of depolarization may be slow. This line of reasoning suggests an observational test. Namely, the rates of depolarization should be correlated with the relative frequency of occurrence of the orthogonal modes. Pulsars with large rates of depolarization should have modes which occur with nearly equal frequency. Conversely, the radio emission from pulsars with small rates of depolarization should be dominated by a single mode. To investigate the proposed observational test in more detail, consider two specific examples: PSR B0834+06, which depolarizes faster than any other 
object in the sample of Morris et al. (1981), and PSR B1929+10, which has a relatively flat depolarization index.

The frequency of occurrence of the modes is evident in observations of individual pulse polarization. Stinebring et al. (1984) reported the results of their single pulse observations in the form of grey-scale histograms. The histograms show how circular polarization, position angle, and linear polarization fluctuate across a pulsar's pulse. In the case of $0834+06$ (Figure 8 of Stinebring et al.), orthogonal modes are clearly obvious in the position angle display. As expected from the large depolarization index of the pulsar, the modes occur with nearly equal frequency across the entire pulse profile. For 1929+10 (Figures 22 and 23 of Stinebring et al.), orthogonal modes are again obvious. However, the position angle display is dominated by a single mode of polarization, which explains this pulsar's small depolarization index. So, to answer the question originally posed, it appears that pulsars have different rates of depolarization because the relative strength of the orthogonal modes, as determined from their relative frequency of occurrence, varies from pulsar-to-pulsar.

\section{Summary}

Both the broadening and the depolarization of pulsar average profiles have been attributed to a single propagation effect: the birefringence of the plasma above pulsar polar caps. The spectra of fractional linear polarization and pulse width are qualitatively consistent with this hypothesis. The observation that orthogonal modes are superposed is also consistent with birefringence. The apparent correlation between depolarization index and relative frequency of occurrence of the modes suggests that pulsars depolarize via superposed modes. data.

Acknowledgments. I thank S. E. Thorsett for sharing his pulse width

\section{References}

Allen, M. C. \& Melrose, D. B. 1982, Proc. Astron. Soc. Aust., 4, 365

Backer, D. C. 1976, ApJ, 209, 895

Barnard, J. J. \& Arons, J. 1986, ApJ, 302, 138

Cocke, W. J. \& Pacholczyk, A. G., 1976, ApJ, 204, L13.

Malofeev, V. M. \& Malov, I. F., 1980, Sov. Astr., 24, 54

Manchester, R. N., Taylor, J. H., \& Huguenin, G. R., 1975, ApJ, 196, 83

McKinnon, M. M., 1996, ApJ, submitted

Melrose, D. B. \& Stoneham, R. J., 1977, Proc. Astron. Soc. Aust., 3, 120

Melrose, D. B., 1979, Aust. J. Phys., 32, 61

Morris, D., Graham, D. A., \& Sieber, W., 1981, A\&A, 100, 107

Phillips, J. A. \& Wolszczan, A. 1992, ApJ, 385, 273

Stinebring, D. R., Cordes, J. M., Rankin, J. M., Weisberg, J. M., \& Boriakoff, V., 1984, ApJS, 55, 247

Thorsett, S. E., 1991, ApJ, 377, 263 